Красноярский государственный медицинский университет имени профессора В.Ф. Войно-Ясенецкого, Красноярск, Россия

${ }^{2}$ Краевая клиническая больница, Красноярск, Россия

${ }^{3}$ Красноярский научный центр Сибирского отделения Российской академии наук, обособленное подразделение «НИИ медицинских проблем Севера», Красноярск, Россия

ОБОСНОВАНИЕ. Эффективный контроль аутоиммунного воспаления при болезни Грейвса предопределяет необходимость изучения дисфункции хелперных и цитотоксических Т-лимфоцитов, а также степени активации регуляторных Т-клеток при тиреостатической терапии болезни Грейвса, что позволит уточнить иммуномодулирующие эффекты длительной консервативной терапии тиамазолом и определить мишени для разработки современной таргетной терапии.

ЦЕЛЬ. Изучить фенотипический состав Т-лимфоцитов периферической крови у пациентов с болезнью Грейвса для оценки направленности иммунного ответа в зависимости от длительности медикаментозного эутиреоза.

МАТЕРИАЛЫ И МЕТОДЫ. Проведено одноцентровое одномоментное когортное сплошное открытое контролируемое исследование с оценкой фенотипического состава Т-клеток в периферической крови у женщин с болезнью Грейвса. Методом проточной цитометрии с использованием прямой иммунофлуоресценции с применением моноклональных антител были исследованы особенности фенотипа Т-лимфоцитов в зависимости от продолжительности медикаментозного эутиреоза при консервативной терапии тиамазолом.

РЕзУЛЬтАТЫ. В исследование включены 135 женщин с верифицированным диагнозом болезни Грейвса, средний

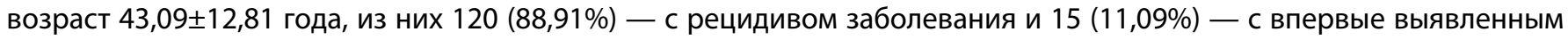
гипертиреозом. Установлено повышение процентного содержания активированных T-хелперов $\left(\mathrm{CD} 3^{+} \mathrm{CD} 4^{+} \mathrm{CD} 25^{+}\right)$ у больных болезнью Грейвса с продолжительностью медикаментозного эутиреоза от 5 до 8 мес и от 9 до 12 мес соответственно, Ме=0,94 (0,48-1,45; $p=0,020)$ и $\mathrm{Me}=0,95(0,41-1,80 ; p=0,025)$, у лиц контрольной группы - Ме=0,12 $(0,03-0,68)$. Установлено повышение количества регуляторных Т-лимфоцитов (CD4+CD25+CD127 $\left.{ }^{\text {Low }}\right)$ как в группе больных с продолжительностью медикаментозного эутиреоза от 5 до 8 мес (Me=3,01 $(1,88-4,47) ; p=0,024)$, так и у пациентов с длительностью медикаментозного эутиреоза от 9 до 12 мес (Me=5,52 (2,77-11,61); p<0,001) в сравнении с показателями группы контроля (Ме=1,81 (0,91-2,82)). Уровень регуляторных Т-клеток в периферической крови пациентов с болезнью Грейвса с продолжительностью медикаментозного эутиреоза более 12 мес снижается, но сохраняется повышенным относительно контроля.

ЗАКЛюЧЕНИЕ. У пациентов с болезнью Грейвса с продолжительностью медикаментозного эутиреоза от 5 до 8 мес и от 9 до 12 мес повышается популяция регуляторных Т-лимфоцитов с фенотипом CD4+CD25+CD127 Low. Koличество активированных Т-хелперов с фенотипом $\mathrm{CD} 3^{+} \mathrm{CD} 4^{+} \mathrm{CD} 25^{+}$сохраняется повышенным независимо от продолжительности медикаментозного эутиреоза. У пациентов с болезнью Грейвса с продолжительностью медикаментозного эутиреоза более 12 мес сохраняется компенсаторное повышение регуляторных Т-лимфоцитов, а общее количество Т-хелперов восстанавливается до уровня контроля.

КЛЮЧЕВЫЕ СЛОВА: болезнь Грейвса; Т-регуляторные клетки; активированные Т-хелперы; иммуномодулирующие эффректы тиреостатических препаратов; таргетная терапия.

\title{
T-LYMPHOCYTES PHENOTYPIC COMPOSITION OF PERIPHERAL BLOOD IN PATIENTS WITH GRAVES' DISEASE UNDERGOING CONSERVATIVE THERAPY WITH THIAMAZOLE
}

(c) Margarita A. Dudina ${ }^{1,2 *}$, Sergey A. Dogadin ${ }^{1,2}$, Andrei A. Savchenko ${ }^{3}$, Vasiliy D. Belenyuk ${ }^{3}$

${ }^{1}$ Krasnoyarsk State Medical University, Krasnoyarsk, Russia

${ }^{2}$ Krasnoyarsk regional clinical hospital, Krasnoyarsk, Russia

${ }^{3}$ Federal Research Center «Krasnoyarsk Science Center» of the Siberian Branch of the Russian Academy of Sciences,

Scientific Research Institute of medical problems of the North, Laboratory of Molecular and Cell physiology and pathology, Krasnoyarsk, Russia 
BACKGROUND: Effective control of autoimmune inflammation in Graves' disease determines necessity to study the Thelper (Th) and cytotoxic T-lymphocytes dysfunction, as well as the level of regulatory T-cells (Treg) activation in patients with Graves' disease on thyrostatic medication, which will clarify the immunomodulatory effects of long-term thiamazole treatment serve as targets for more specific therapies.

AIM: To study the phenotypic composition of T-lymphocytes in the peripheral blood of patients with Graves' disease to assess the direction of immune response depending on thimazole-induced euthyroidism duration.

MATERIALS AND METHODS: A single-center, cohort, continuous, open-label, controlled trial was conducted to assess the phenotypic composition of T-lymphocytes in peripheral blood in women with Graves' disease on long-term thiamazole treatment. The phenotypic composition of T-lymphocytes was determined by flow cytometry using direct immunofluorescence with conjugated FITC monoclonal antibodies depending on the duration of thimazole-induced euthyroidism of long-term thiamazole treatment.

RESULTS: The study included 135 women with Graves' disease, mean age $43.09 \pm 12.81$ years, 120 (88.91\%) with a relapse of the disease and 15 (11.09\%) with newly diagnosed hyperthyroidism. An increase of activated $\mathrm{CD} 3^{+} \mathrm{CD} 4^{+} \mathrm{CD} 25^{+}$was found in patients with Graves' disease with a duration of thimazole-induced euthyroidism 5-8 months and 9-12 months, respectively, $\mathrm{Me}=0.94(0.48-1.45), \mathrm{p}=0.020)$ and $\mathrm{Me}=0.95(0.41-1.80), \mathrm{p}=0.025)$, in control group $-\mathrm{Me}=0.12(0.03-0.68)$. Compared to the control an increase of $\mathrm{CD} 4{ }^{+} \mathrm{CD} 25^{+} \mathrm{CD} 127^{\text {Low }}$ (Treg) was found in patients with a duration of thimazole-induced euthyroidism 5-8 and 9-12 months. The content of Treg in peripheral blood in Graves' disease patients with a duration of thimazole-induced euthyroidism more than 12 months decreases, but remains elevated relative to the control.

CONCLUSION: In patients with Graves' disease with a duration of thimazole-induced euthyroidism 5-8 months and 9-12 months the level of Treg has been increased. The increase of activated Th $\left(C D 3^{+} C D 4^{+} C D 25^{+}\right)$persists independently of thimazole-induced euthyroidism. In patients with Graves' disease with a duration of thimazole-induced euthyroidism for more than 12 months, there is a compensatory increase in regulatory T-lymphocyte, and the total number of T-helpers is restored to the control.

KEYWORDS: Graves' disease; regulatory T-cells; activated T-helpers; immunomodulatory effects of thiamazole; targeting therapies.

\section{ОБОСНОВАНИЕ}

Болезнь Грейвса характеризуется развитием тиреотоксикоза, вызванного циркуляцией антител к рецептору тиреотропного гормона (рТТГ), которые усиливают индуцируемую интерфероном-гамма (ИФН-ү) экспрессию молекул 2-го класса гистосовместимости (HLA-DR) и презентацию тиреоидных антигенов, способствуя дальнейшей активации Т-хелперов (Th), что клинически может проявляться как стимуляцией, так и ингибированием функции щитовидной железы [1]. В последнее время изменился характер течения гипертиреоза аутоиммунного генеза. Отмечаются выраженное ускорение манифестации заболевания у предрасположенных лиц, резкое снижение количества пациентов с ремиссией тиреотоксикоза и ее продолжительностью на фоне консервативной терапии тиреостатиками [2].

Механизм иммуносупрессивного действия тиреостатических препаратов остается предметом споров, несмотря на многочисленные утверждения о том, что тионамиды напрямую ингибируют йодирование тиреоглобулина, что может повлиять на презентацию антигенов тиреоцитами, а также препятствуют экспрессии в тиреоцитах таких молекул, как интерлейкин-1 (IL-1), интерлейкин-6 (IL-6), простагландин E2 и белок теплового шока [3]. Однако в ряде клинических исследований у пациентов с болезнью Грейвса отмечаются снижение активации CD4+ Т-лимфоцитов и повышение количества $\mathrm{CD}^{+}$цитотоксических Т-лимфоцитов, а также понижение уровня растворимых рецепторов IL-2, что определяется достижением стойкого медикаментозного эутиреоза при длительной тионамидной терапии метимазолом [4, 5].
В настоящее время имеются предположения о том, что Т-хелперный иммунный ответ 1-го типа (Th1) преобладает в иммунопатогенезе болезни Грейвса во время активной фазы гипертиреоза, в которой хемокины Th1 и их рецептор CXCR3 играют ключевую роль [6]. Эти нарушения считаются результатом первичного нарушения иммунорегуляции, при которой дисфункция щитовидной железы обуславливается агрессией сохранивших активность Th1- и Th2-лимфоцитов против специфических антигенов клеток-мишеней. Остаются до сих пор неизвестными влияния тиреостатической терапии на дефект недостаточной активации регуляторных Т-клеток и формирование супрессорных механизмов в иммунной системе при болезни Грейвса, что не только определяет степень дисфункции тиреоидспецифических хелперных Т-лимфоцитов, но и может непосредственно индуцировать переход от доминирования Th2- к доминированию Th1-иммунного ответа в клиническом течении заболевания.

В связи с этим эффективный контроль аутоиммунного воспаления при болезни Грейвса предопределяет необходимость уточнения иммунологических критериев прогрессирования заболевания на фоне тиреостатической терапии для разработки современной стратегии таргетной терапии.

\section{ЦЕЛЬ ИССЛЕДОВАНИЯ}

Изучить фенотипический состав Т-лимфоцитов периферической крови у пациентов с болезнью Грейвса для оценки направленности иммунного ответа в зависимости от длительности медикаментозного эутиреоза. 


\section{МАТЕРИАЛЫ И МЕТОДЫ}

\section{Место и время проведения исследования}

Место проведения. Исследование проводилось на базе эндокринологического центра КГБУЗ «Краевая клиническая больница» (г. Красноярск).

Время исследования. Наблюдение пациентов осуществлялось с 9 апреля 2016 г. по 10 апреля 2021 г.

Изучаемые популяции (одна или несколько)

Изучались две популяции: пациенты с болезнью Грейвса и здоровые индивиды (контроль).

\section{Популячия «пациенты с болезнью Грейвса»}

Критерии включения: женский пол, возраст от 18 до 65 лет, лабораторно подтвержденная болезнь Грейвса, стойкий медикаментозный эутиреоз, индуцированный непрерывным приемом тиамазола.

\section{Популячия «контроль»}

Критерии включения: женский пол, возраст от 18 до 65 лет, отсутствие отягощенного анамнеза по заболеваниям щитовидной железы у обследуемой пациентки и кровных родственников, отсутствие структурных изменений по данным ультразвукового исследования (УЗИ) щитовидной железы.

Критерии исключения: узловой/многоузловой токсический зоб, беременность, лактация, эндокринная офтальмопатия, рецидив гипертиреоза после лечения болезни Грейвса радиоактивным йодом, наличие инфекционных и аллергических заболеваний, новообразования, системные заболевания соединительной ткани, другие заболевания органов эндокринной системы, острые респираторные и вирусные инфекции, а также введение профилактических прививок в течение 2 мес, предшествующих иммунологическому и гормональному анализу.

\section{Способ формирования выборки из изучаемой}

популяции (или нескольких выборок из нескольких изучаемых популяций)

В основу формирования подгрупп пациентов с болезнью Грейвса была положена концепция иммуномодулирующего эффекта тиреостатической терапии при условии стойкого медикаментозного эутиреоза, индуцированного непрерывным приемом тиамазола, использовавшегося по стандартному протоколу консервативного лечения болезни Грейвса [7]. Выбор сроков для клинико-иммунологического обследования пациентов с болезнью Грейвса базировался на стабильности достигнутого медикаментозного эутиреоза. Стойкая нормализация гормонов щитовидной железы включала, в том числе, стабилизацию уровня ТТГ в пределах нормального референсного диапазона. Предварительный анализ показал, что сроки поддержания состояния стойкого медикаментозного эутиреоза на фоне непрерывного приема тиамазола у обследуемых пациентов с болезнью Грейвса варьируют от 5 до 12 мес и более. В связи с этим пациентки со стойким медикаментозным эутиреозом при болезни Грейвса были разделены на репрезентативные подгруппы внутри указанного периода наблюдения, а также с учетом рекомендуе- мых сроков консервативного лечения заболевания согласно национальным клиническим рекомендациям. Изучаемая популяция пациентов с болезнью Грейвса была разделена на три группы: первая - продолжительность медикаментозного эутиреоза от 5 до 8 мес, вторая - продолжительность медикаментозного эутиреоза от 9 до 12 мес, третья - продолжительность медикаментозного эутиреоза более 12 мес.

\section{Дизайн исследования}

Проведено одноцентровое одномоментное когортное сплошное открытое контролируемое исследование с ретроспективным анализом данных с участием пациентов с лабораторно подтвержденной болезнью Грейвса.

\section{Методы}

Клинические методы обследования на момент рандомизации включали объективный осмотр, пальпацию щитовидной железы, клинико-лабораторную оценку тиреоидного статуса и титра антител к рТТГ в сыворотке крови. Изучение клинико-анамнестических данных обследуемых пациентов с впервые выявленным или рецидивирующим течением заболевания проводилось с использованием медицинской документации (амбулаторные карты, форма № 025/у-04; истории болезней стационарного больного, форма № 003/y).

При этом оценивали хронологию развития заболевания с уточнением времени от момента манифестации гипертиреоза, верификации диагноза болезни Грейвса и инициации тиреостатической терапии, дозу тиамазола, гормональные показатели, титр антител к рТТГ исходно и на фоне медикаментозного лечения, а также продолжительность тиреостатической терапии. Методом хемилюминесцентного иммуноанализа на микрочастицах на автоматическом анализаторе Architecti1000sr (Abbott Diagnostics, США) определяли уровень ТТГ и свободного тироксина (св.Т4) в сыворотке крови, указанные референсные диапазоны соответственно 0,4-4,0 мЕд/л и 9,01-19,05 пмоль/л. Определение свободного трийодтиронина (св.Т3) в сыворотке крови осуществляли методом энзим-связанного иммуноферментного анализа (ИФА) с использованием тест-систем «ДС-ИФА-ТИРОИД-ТЗсвободный» (ООО «НПО «Диагностические системы», Россия), референсный интервал 2,14-6,42 пмоль/л. Уровень антител к рТТГ оценивался методом ИФА при помощи стандартного набора Medizym T.R.A. (MedipanDiagnostica, Германия), рекомендованная точка разделения (cut-off) - 1,5 мЕд/л («серая» зона 1-1,5 мЕд/л). Уровень антител к тиреоидной пероксидазе (ТПО) оценивался методом ИФА при помощи соответствующего набора «АТ-ТПОХема-Медика» (Россия), референсный интервал $<35$ мЕд/л. Определение размеров, объема и структуры щитовидной железы проводилось на основании УзИ на аппарате Aloka 3500 (Hitachi, Япония) с использованием линейного датчика с частотой 7,5 МГц.

Исследование фенотипа Т-лимфоцитов проводили методом проточной цитометрии с использованием прямой иммунофлуоресценции с применением моноклональных антител (Beckman Coulter, США), меченных FITC (fluoresceinisothiocyanate), PE (phycoerythrin), ECD 
(phycoerythrin-TexasRed-X), PC5 (phycoerythrin-cyanin 5), PC7 (phycoerythrin-cyanin 7) и APC (allophycocyanin) В следующей панели: CD45-FITC/CD127-PE/CD3-ECD/ CD25-PC5/CD4-PC7/CD8-APC. Анализ окрашенных клеток проводили на проточном цитофлуориметре Navios (Beckman Coulter, USA) Центра коллективного пользования КНЦ СО РАН. Обработку полученных цитофлуориметрических результатов осуществляли с помощью программ Navios Software v. 1.2 и Kaluza v. 2.1.1 (Beckman Coulter, USA). В каждой пробе анализировали не менее 50000 лимфоцитов.

\section{Статистический анализ}

Описание полученных данных производили с помощью подсчета медианы (Ме) и квартильного размаха в виде 1 и 3 квартилей $\left(Q_{1}-Q_{3}\right)$, а также в виде средних арифметических значений и стандартных отклонений от среднего $(\mathrm{M} \pm \mathrm{SD})$ в случае нормального распределения показателей. Для определения характера распределения полученных данных использовали критерий Шапиро-Уилка. Достоверность различий между исследуемыми показателями оценивали по непараметрическому критерию Манна-Уитни (Mann-Whitney U-test). Для исследования силы взаимосвязей показателей вычислялся коэффициент ранговой корреляции по Спирмену (Spearman rank R). Статистический анализ осуществляли в пакете прикладных программ Statistica 8.0 (StatSoft Inc., 2007).

\section{Этическая экспертиза}

Протокол исследования одобрен Локальным этическим комитетом КГБУЗ «Краевая клиническая больница» от 07 апреля 2016 г. (выписка из протокола № 124) и ФБОУ ВО «КрасГМУ» от 9 ноября 2016 г. (Выписка из протокола № 72/2016).

\section{РЕЗУЛЬТАТЫ}

В исследование были включены 135 женщин с верифицированным диагнозом болезни Грейвса, средний возраст 43,14士12,84 года, из них 120 (88,9\%) - с рецидивом заболевания и $15(11,1 \%)$ - с впервые выявленным гипертиреозом. В качестве контроля обследованы 85 неродственных, практически здоровых женщин.

Все обследованные пациенты с болезнью Грейвса наблюдались в исследовательском центре с дебюта заболевания. При ретроспективном анализе показателей тиреоидного статуса в дебюте заболевания было установлено, что у обследованных пациентов с болезнью Грейвса I и II групп был диагностирован субклинический или манифестный гипертиреоз соответственно, ТТГ=0,07 мЕд/л (0,03-0,39), св.Т3=5,92 пмоль/л (4,61-6,41), св.Т4=17,33 пмоль/л (12,19-18,77) и ТТГ=0,05 мЕд/л $(0,01-$ 0,37), св.Т3=6,51 пмоль/л (4,31-7,43), св.Т4=21,52 пмоль/л (19,11-36,77). У большинства пациентов III группы показатели тиреоидного статуса в дебюте заболевания соответствовали субклиническому гипертиреозу: ТТГ=0,03 мЕд/л $(0,09-0,36)$, св.Т3=5,12 пмоль/л $(3,92-$ 6,31), св.Т4=16,41 пмоль/л (12,88-18,78). При ретроспективном сравнительном анализе исходных показателей тиреоидного статуса и уровня антител к рТТГ подгрупп пациентов с болезнью Грейвса, в дальнейшем обследованных в зависимости от продолжительности медика- ментозного эутиреоза, статистически значимых различий не выявлено.

Независимо от продолжительности медикаментозного эутиреоза в периферической крови обследуемых пациентов с болезнью Грейвса в дебюте заболевания был повышен относительный уровень Т-хелперов $\left(\mathrm{CD} 3^{+} \mathrm{CD} 4^{+}\right)$, в том числе активированных $\left(\mathrm{CD}^{+}{ }^{+} \mathrm{CD} 4^{+} \mathrm{CD} 25^{+}\right)$соответственно, Ме=66,42\% (44,23-74,61), $\mathrm{p}=0,011$ и $\mathrm{Me}=5,51 \%$ $(4,12-6,73), p=0,013$. Процентное количество регуляторных $\mathrm{CD}^{+}{ }^{+} \mathrm{CD} 4^{+} \mathrm{CD} 127^{\text {Low }} \mathrm{CD} 25^{\text {High }}$-клеток в крови у всех обследуемых пациентов на момент манифестации болезни Грейвса было снижено - Ме=1,21\% (0,92-1,81), p=0,021.

Клинико-гормональная характеристика обследуемых пациентов с болезнью Грейвса на момент включения в настоящее исследование представлена в таблице 1.

На момент клинико-иммунологического обследования у всех пациентов был достигнут индуцированный тиамазолом медикаментозный эутиреоз, что подтверждалось при объективном осмотре и соответствующими показателями тиреоидного статуса. Все обследуемые пациенты с болезнью Грейвса имели положительный титр антител к рТТГ, причем величина вышеуказанного маркера не различалась в зависимости от продолжительности медикаментозного эутиреоза. При этом максимальная продолжительность приема тиамазола в III группе больных составила 36 мес.

При исследовании фенотипа Т-лимфоцитов было обнаружено, что у пациентов с болезнью Грейвса абсолютное и относительное содержание Т-клеток $\left(\mathrm{CD}^{+}\right)$ в периферической крови не изменялось в зависимости от продолжительности медикаментозного эутиреоза и соответствовало контрольным значениям (табл. 2).

При сравнительном анализе фенотипического состава Т-лимфоцитов периферической крови в зависимости от продолжительности медикаментозного эутиреоза наиболее выраженные изменения обнаружены в увеличении содержания Т-клеток с фенотипом $\mathrm{CD}^{+} \mathrm{CD}_{25}{ }^{+}$, несущих маркер ранней активации. С увеличением интервала времени нахождения пациентов с болезнью Грейвса в состоянии медикаментозного эутиреоза количество активированных Т-лимфоцитов (CD3+CD25+) последовательно повышалось. Относительное количество Т-хелперов $\left(\mathrm{CD}^{+} \mathrm{CD}^{+}\right)$у пациенток с болезнью Грейвса и медикаментозным эутиреозом в течение 5-8 мес было увеличено в сравнении с контрольным диапазоном. При увеличении продолжительности медикаментозного эутиреоза уровень $\mathrm{CD}^{+}{ }^{+} \mathrm{CD} 4^{+}$-клеток стал соответствовать контрольным значениям. А при длительности эутиреоза более 12 мес содержание Т-хелперов было значительно ниже, чем в I группе. Уровень цитотоксических Т-лимфоцитов $\left(\mathrm{CD}^{+}{ }^{+} \mathrm{CD} 8^{+}\right)$был значительно ниже контрольных значений как в группе пациентов с продолжительностью медикаментозного эутиреоза 5-8 мес, так и 9-12 мес, тогда как в III группе больных (эутиреоз более 12 мес) содержание цитотоксических Т-клеток соответствовало контрольному диапазону. Относительное количество активированных T-хелперов $\left(\mathrm{CD}^{+}{ }^{+} \mathrm{CD} 4^{+} \mathrm{CD} 25^{+}\right)$было повышено у пациентов с продолжительностью медикаментозного эутиреоза в течение 5-8 и 9-12 мес и еще более возрастало в группе больных с продолжительностью медикаментозного эутиреоза более 12 мес. 
Таблица 1. Клинико-гормональная характеристика пациентов с болезнью Грейвса по группам, $\mathrm{Me}\left(\mathrm{Q}_{1}-\mathrm{Q}_{3}\right)$

\begin{tabular}{|c|c|c|c|c|}
\hline \multirow{3}{*}{ Показатели } & \multirow{2}{*}{$\begin{array}{c}\text { Контроль } \\
\text { n=85 }\end{array}$} & \multicolumn{3}{|c|}{ Пациенты с болезнью Грейвса } \\
\hline & & $\begin{array}{c}\text { Группа I } \\
\text { Медикамент. } \\
\text { эутиреоз } \\
\text { 5-8 мес } \\
\text { n=41 }\end{array}$ & $\begin{array}{c}\text { Группа II } \\
\text { Медикамент. } \\
\text { эутиреоз } \\
\text { 9-12 мес } \\
\text { n=69 }\end{array}$ & $\begin{array}{c}\text { Группа III } \\
\text { Медикамент } \\
\text { эутиреоз } \\
>12 \text { мес } \\
\text { n=25 }\end{array}$ \\
\hline & 1 & 2 & 3 & 4 \\
\hline
\end{tabular}

Возраст, лет $(\mathrm{M} \pm \mathrm{SD})$

$41,02 \pm 12,11$

$43,91 \pm 13,76$

$42,94 \pm 12,24$

$42,43 \pm 13,09$

Продолжительность приема тиамазола, мес
7,0

$(6,0-8,0)$

10,0

(10-20)
11,0

$(10,0-12,0)$
16,0

$(14,0-24,0)$

Доза тиамазола, мг

$\begin{array}{cc}- & 10,0 \\ - & (10-20)\end{array}$

10,0

(10-20)

10,0

(10-15)

\begin{tabular}{|c|c|c|c|c|}
\hline $\begin{array}{l}\text { Тиреотропный гормон, } \\
\text { мЕд/л }\end{array}$ & $\begin{array}{c}1,13 \\
(0,86-1,51)\end{array}$ & $\begin{array}{c}0,78 \\
(0,41-2,11) \\
p_{1}<0,001\end{array}$ & $\begin{array}{c}1,09 \\
(0,55-3,43) \\
p_{1}<0,001\end{array}$ & $\begin{array}{c}1,91 \\
(0,57-5,31) \\
p_{1}=0,004\end{array}$ \\
\hline Св.ТЗ, пмоль/л & $\begin{array}{c}4,07 \\
(2,61-5,53)\end{array}$ & $\begin{array}{c}5,31 \\
(3,83-6,31) \\
p_{1}<0,001\end{array}$ & $\begin{array}{c}4,73 \\
(3,82-5,93) \\
p_{1}<0,001\end{array}$ & $\begin{array}{c}2,24 \\
(2,93-5,12) \\
p_{1}=0,027\end{array}$ \\
\hline Св.Т4, пмоль/л & $\begin{array}{c}14,10 \\
(12,28-15,80)\end{array}$ & $\begin{array}{c}13,07 \\
(10,49-16,91) \\
\mathrm{p}_{1}<0,001\end{array}$ & $\begin{array}{c}12,83 \\
(10,19-16,97) \\
p_{1}<0,001\end{array}$ & $\begin{array}{c}15,57 \\
(11,83-17,43) \\
p_{1}=0,022 \\
p_{3}=0,030\end{array}$ \\
\hline Антитела к рТТГ, мЕд/л & $\begin{array}{c}0,24 \\
(0,18-0,43)\end{array}$ & $\begin{array}{c}15,03 \\
(9,45-21,04) \\
p_{1}<0,001\end{array}$ & $\begin{array}{c}15,27 \\
(9,45-21,45) \\
p_{1}<0,001\end{array}$ & $\begin{array}{c}15,62 \\
(9,32-24,52) \\
p_{1}<0,001\end{array}$ \\
\hline Антитела к ТПО, мЕд/л & $\begin{array}{c}1,00 \\
(0,00-3,00)\end{array}$ & $\begin{array}{c}223,21 \\
(13,01-563,11) \\
p_{1}<0,001\end{array}$ & $\begin{array}{c}227,21 \\
(29,13-695,51) \\
p_{1}<0,001\end{array}$ & $\begin{array}{c}632,00 \\
(78,00-1186,00) \\
p_{1}=0,003\end{array}$ \\
\hline $\begin{array}{l}\text { Объем щитовидной } \\
\text { железы, мл }\end{array}$ & $\begin{array}{c}9,91 \\
(9,41-12,63)\end{array}$ & $\begin{array}{c}29,19 \\
(20,41-45,32) \\
p_{1}<0,001\end{array}$ & $\begin{array}{c}32,21 \\
(20,51-49,88) \\
p_{1}<0,001\end{array}$ & $\begin{array}{c}27,36 \\
(16,65-50,61) \\
p_{1}<0,001\end{array}$ \\
\hline
\end{tabular}

Примечание. $\mathrm{p}_{1}$ - статистически значимые различия с контрольными величинами; $\mathrm{p}_{2}-$ статистически значимые различия с показателями пациентов І-й группы; $p_{3}$ - статистически значимые различия с показателями пациентов II-й группы; св.Т4 - свободный тироксин; св.Т3 - свободный трийодтиронин; РТТГ — рецептор тиреотропного гормона; ТПО — тиреоидная пероксидаза.

У пациентов I группы процентное содержание в крови Т-клеток с фенотипом $\mathrm{CD}^{+} \mathrm{CD}^{+} \mathrm{CD} 25^{+}$соответствовало контрольным значениям, а в III группе их уровень был значительно выше контрольного. В то же время в III группе (эутиреоз более 12 мес) количество активированных Т-хелперов $\left(\mathrm{CD}^{+} \mathrm{CD}^{+} \mathrm{CD} 25^{+}\right)$было значительно выше как относительно контрольных значений, так и по сравнению с данными, выявляемыми у пациентов I и II групп (продолжительность эутиреоза 5-8 и 9-12 мес).
Относительное количество Т-регуляторных клеток $\left(\mathrm{CD} 3^{+} \mathrm{CD} 4^{+} \mathrm{CD} 127^{\text {Low }} \mathrm{CD} 25^{\text {High }}\right)$ в периферической крови у пациентов с болезнью Грейвса в сравнении с контрольным диапазоном было повышено независимо от продолжительности медикаментозного эутиреоза. Однако у больных с продолжительностью эутиреоза более 12 мес наблюдалось снижение содержания Т-регуляторных клеток относительно уровня, выявленного при медикаментозном эутиреозе продолжительностью 9-12 мес. 
Таблица 2. Фенотипический состав Т-лимфоцитов в периферической крови пациентов с болезнью Грейвса (Ме, (Q1-Q3)

\begin{tabular}{|c|c|c|c|c|}
\hline \multirow{3}{*}{ Показатели } & \multirow{2}{*}{$\begin{array}{c}\text { Контроль } \\
\text { n=85 }\end{array}$} & \multicolumn{3}{|c|}{ Пациенты с болезнью Грейвса } \\
\hline & & $\begin{array}{c}\text { Группа I } \\
\text { Медикамент. } \\
\text { эутиреоз } \\
\text { 5-8 мес } \\
\text { n=41 }\end{array}$ & $\begin{array}{c}\text { Группа II } \\
\text { Медикамент. } \\
\text { эутиреоз } \\
9-12 \text { мес } \\
\text { n=69 }\end{array}$ & $\begin{array}{c}\text { Группа III } \\
\text { Медикамент. } \\
\text { эутиреоз } \\
>12 \text { мес } \\
\text { n=25 }\end{array}$ \\
\hline & 1 & 2 & 3 & 4 \\
\hline $\mathrm{CD}^{+}, 10^{9} / л$ & $\begin{array}{c}1,51 \\
(1,19-1,65)\end{array}$ & $\begin{array}{c}2,00 \\
(1,14-2,52)\end{array}$ & $\begin{array}{c}1,59 \\
(1,17-2,03)\end{array}$ & $\begin{array}{c}1,81 \\
(0,63-2,29)\end{array}$ \\
\hline $\mathrm{CD}^{+}, \%$ & $\begin{array}{c}72,0 \\
(68,31-76,12)\end{array}$ & $\begin{array}{c}77,6 \\
(69,21-84,62)\end{array}$ & $\begin{array}{c}74,41 \\
(66,81-78,41)\end{array}$ & $\begin{array}{c}70,95 \\
(58,91-75,49)\end{array}$ \\
\hline $\mathrm{CD}^{+}{ }^{+} \mathrm{CD} 25^{+}, \%$ & $\begin{array}{c}0,21 \\
(0,03-1,70)\end{array}$ & $\begin{array}{c}1,18 \\
(0,33-3,07) \\
p_{1}=0,045\end{array}$ & $\begin{array}{c}1,41 \\
(0,66-2,52) \\
p_{1}=0,040 \\
p_{2}=0,027\end{array}$ & $\begin{array}{c}2,67 \\
(1,74-4,02) \\
\mathrm{p}_{1}<0,001 \\
\mathrm{p}_{2}=0,038 \\
\mathrm{p}_{3}=0,043\end{array}$ \\
\hline $\mathrm{CD}^{+}{ }^{+} \mathrm{CD}^{+}, \%$ & $\begin{array}{c}41,4 \\
(37,8-46,2)\end{array}$ & $\begin{array}{c}52,7 \\
(38,4-55,4) \\
p_{1}=0,031\end{array}$ & $\begin{array}{c}49,7 \\
(39,9-54,4)\end{array}$ & $\begin{array}{c}40,1 \\
(34,4-48,3) \\
p_{2}=0,042\end{array}$ \\
\hline $\mathrm{CD}^{+} \mathrm{CD}^{+}, \%$ & $\begin{array}{c}30,1 \\
(26,2-35,1)\end{array}$ & $\begin{array}{c}25,2 \\
(21,5-26,2) \\
p_{1}=0,014\end{array}$ & $\begin{array}{c}22,3 \\
(20,1-28,7) \\
p_{1}<0,001\end{array}$ & $\begin{array}{c}29,3 \\
(18,3-36,3)\end{array}$ \\
\hline $\mathrm{CD}^{+}{ }^{+} \mathrm{CD}^{+}{ }^{+} \mathrm{CD}_{25} 5^{+} \%$ & $\begin{array}{c}0,12 \\
(0,03-0,68)\end{array}$ & $\begin{array}{c}0,94 \\
(0,48-1,45) \\
p_{1}=0,020\end{array}$ & $\begin{array}{c}0,95 \\
(0,41-1,80) \\
p_{1}=0,025\end{array}$ & $\begin{array}{c}1,65 \\
(0,78-2,97) \\
\mathrm{p}_{1}<0,001 \\
\mathrm{p}_{2}=0,036 \\
\mathrm{p}_{3}=0,038\end{array}$ \\
\hline $\mathrm{CD}^{+}{ }^{+} \mathrm{CD}^{+}{ }^{+} \mathrm{CD}_{25} 5^{+} \%$ & $\begin{array}{c}0,06 \\
(0,03-0,17)\end{array}$ & $\begin{array}{c}0,31 \\
(0,03-1,05)\end{array}$ & $\begin{array}{c}0,33 \\
(0,12-0,94) \\
p_{1}=0,045\end{array}$ & $\begin{array}{c}0,99 \\
(0,52-1,88) \\
\mathrm{p}_{1}<0,001 \\
\mathrm{p}_{2}=0,043 \\
\mathrm{p}_{3}=0,032\end{array}$ \\
\hline $\mathrm{CD}^{+}{ }^{+} \mathrm{CD}^{+}{ }^{+} \mathrm{CD} 127^{\text {Low }} \mathrm{CD} 25^{\text {High }}, \%$ & $\begin{array}{c}1,81 \\
(0,91-2,82)\end{array}$ & $\begin{array}{c}3,01 \\
(1,88-4,47) \\
p_{1}=0,024\end{array}$ & $\begin{array}{c}5,52 \\
(2,77-11,61) \\
\mathrm{p}_{1}<0,001\end{array}$ & $\begin{array}{c}3,21 \\
(1,04-4,30) \\
p_{1}=0,048 \\
p_{3}=0,039\end{array}$ \\
\hline
\end{tabular}

Примечание. $\mathrm{p}_{1}$ - статистически значимые различия с контрольными величинами; $\mathrm{p}_{2}$ - статистически значимые различия с показателями пациентов I группы; $p_{3}$ - статистически значимые различия с показателями пациентов II-й группы.

\section{ОБСУЖДЕНИЕ}

Эффективность тиреостатической терапии в индукции и поддержании ремиссии болезни Грейвса определяется уровнем экспрессии рТТГ, за счет которых реализуется механизм дальнейшей презентации тиреоидных антигенов и увеличения количества активированных Т-хелперов [8]. Длительная тиреостатическая терапия приводит к снижению титра циркулирующих антител к рТТГ, выраженности лимфоцитарной инфильтрации щитовидной железы, уменьшению антигенной презентации на поверхности фолликулярных клеток и развитию медикаментозного эутиреоза [9]. Однако наблюдение за пациентками с болезнью Грейвса, длительно принимающими тиамазол, показало, что уровень антител к рТТГ остается положительным на любой стадии процесса, независимо от продолжительности тиреостатической терапии, наличия гипертиреоза или медикаментозного эутиреоза, а также не связан с изменениями фенотипического состава Т-лимфоцитов.

Причем необходимо отметить, что у пациентов с болезнью Грейвса в зависимости от продолжительности медикаментозного эутиреоза меняется именно фенотипический состав Т-лимфоцитов, в то время как процентное и абсолютное количество общих Т-клеток в крови остается постоянным и не отличается от контрольных показателей. В то же время у обследованных больных с разной продолжительностью медикаментозного эутиреоза наблюдается определенная динамика изменения относительного количества Т-хелперов $\left(\mathrm{CD}^{+} \mathrm{CD}^{+}\right)$и цитотоксических Т-клеток $\left(\mathrm{CD}^{+}{ }^{+} \mathrm{CD} 8^{+}\right)$. Так, если у пациентов с продолжительностью медикаментозного эутиреоза в течение 5-8 мес в крови выявляются повышенный (относительно контрольных значений) уровень Т-хелперов 
и сниженное количество цитотоксических Т-лимфоцитов, то у больных с продолжительностью медикаментозного эутиреоза 9-12 мес содержание Т-хелперов нормализуется, тогда как количество цитотоксических Т-клеток остается пониженным. При стойком медикаментозном эутиреозе более 12 мес у пациентов с болезнью Грейвса в крови наблюдаются еще более пониженный уровень (относительно исходного, но в диапазоне контрольных значений) Т-хелперов и нормализация количества цитотоксических Т-клеток. Необходимо отметить, что именно в составе субпопуляции Т-хелперов находится фракция Т-регуляторных клеток, которая реализует функции супрессии иммунного ответа [10].

Отмечено, что при болезни Грейвса снижена функциональная активность или количество Т-регуляторных клеток $[1,6]$. В настоящем исследовании показано, что уже при поддержании стойкого медикаментозного эутиреоза в течение 5-8 мес наблюдается иммуномодулирующий эффект консервативной терапии тиамазолом, заключающийся в выраженном увеличении количества Т-регуляторных клеток. При большей длительности медикаментозного эутиреоза (9-12 мес) содержание данной фракции Т-лимфоцитов достигает максимума, тогда как при дальнейшем увеличении длительности медикаментозного эутиреоза (более 12 мес) их количество в крови снова снижается, но сохраняется компенсаторное повышение относительно контроля. Повышение доли регуляторных Т-лимфоцитов $\left(\mathrm{CD}^{+} \mathrm{CD} 4^{+} \mathrm{CD} 127^{\text {Low }} \mathrm{CD} 25^{\text {High }}\right)$ в периферической крови у больных с продолжительностью медикаментозного эутиреоза более 12 мес свидетельствует о том, что длительное лечение тиамазолом способствует однонаправленным изменениям: развитию медикаментозного эутиреоза и частичному восстановлению супрессорного потенциала регуляторных Т-лимфоцитов. Можно предположить, что высокий уровень Т-регуляторных клеток у пациентов с болезнью Грейвса, независимо от стойкости медикаментозного эутиреоза, является компенсаторной иммунной реакцией, направленной на преодоление дефекта резистентности к супрессорному действию регуляторных Т-лимфоцитов, необходимых на любой стадии процесса постоянной антигензависимой активации Т-хелперов при болезни Грейвса.

Кроме оценки субпопуляционного состава Т-лимфоцитов у пациентов с болезнью Грейвса в зависимости от длительности медикаментозного эутиреоза, также было исследовано содержание активированных Т-хелперов и цитотоксических Т-лимфоцитов. В качестве активационного маркера был исследован CD25. Молекула CD25 представляет собой гликопротеин, являющийся низкоаффинным рецептором к интерлейкину-2 и экспрессирующийся на Т-регуляторных клетках, активированных Т-хелперах и цитотоксических Т-лимфоцитах [11]. Количество активированных Т-хелперов (CD3+CD4+CD25+) повышено уже в I группе больных (эутиреоз в течение 5-8 мес). Максимальный уровень Т-клеток с данным фенотипом выявляется во II группе (эутиреоз длительностью более 12 мес). Содержание активированных цитотоксических Т-клеток $\left(\mathrm{CD}^{+}{ }^{+} \mathrm{CD} 8^{+} \mathrm{CD} 25^{+}\right)$повышается у больных II группы (эутиреоз 9-12 мес) и также достигает максимума в III группе пациентов (эутиреоидное состояние в течение более 12 мес) консервативного лечения тиамазолом.
Таким образом, у пациентов с болезнью Грейвса в зависимости от продолжительности медикаментозного эутиреоза меняется фенотипический состав Т-лимфоцитов, но при сохранении абсолютного и процентного содержания общих Т-клеток в периферической крови. Причем изменения фенотипа Т-лимфоцитов при медикаментозном эутиреозе разной продолжительности нельзя однозначно характеризовать как иммуносупрессивные. у больных с наибольшей продолжительностью медикаментозного эутиреоза (более 12 мес) нормализуется количество Т-хелперов и цитотоксических Т-лимфоцитов. Но в то же время у всех обследуемых пациентов с болезнью Грейвса сохраняются характерные изменения начала иммунной реакции с ослаблением супрессии активированных Т-хелперов, не зависящие от дозы и продолжительности медикаментозного эутиреоза. Вероятно, при болезни Грейвса супрессия Т-хелперов ослабляется в такой степени, что они в присутствии антигена выходят из-под контроля и активируются.

Следует подчеркнуть, что активированные Т-хелперы прямо влияют на тиреоциты, продуцируя цитокины, стимулируют цитотоксические клетки, что приводит к повреждению тиреоидной ткани, а также усиливают продукцию плазматическими клетками тиреоидных аутоантител, которые вносят дополнительный вклад в поддержание аутоиммунного воспаления [12]. Тем не менее у пациентов с болезнью Грейвса и продолжительностью медикаментозного эутиреоза от 9 до 12 мес и более общее число Т-хелперов $\left(\mathrm{CD}^{+} \mathrm{CD}^{+}\right)$в периферической крови соответствует контрольным значениям, что свидетельствует о частичном подавлении чрезмерной активации хелперных Т-клеток вместе с увеличением продолжительности времени нахождения пациентов с болезнью Грейвса в состоянии медикаментозного эутиреоза при консервативном лечении тиамазолом.

В ранее проведенных нами исследованиях было показано, что у пациентов с болезнью Грейвса, непрерывно получающих тиамазол от 12 до 18 мес, содержание T-хелперов с фенотипами $\mathrm{CD}^{+}{ }^{+} \mathrm{CD} 4^{+}-$и $\mathrm{CD} 3^{+} \mathrm{CD} 4^{+} \mathrm{CD} 25^{+}$ в периферической крови и ткани щитовидной железы соответствует контрольным значениям, а пониженное относительное число регуляторных Т-лимфоцитов в периферической крови соответствует их уровню в ткани щитовидной железы [13]. В то же время при более продолжительном периоде сохранения состояния медикаментозного эутиреоза в крови увеличивается содержание активированных Т-хелперов и цитотоксических Т-лимфоцитов. Подобный результат изменения фенотипического состава Т-клеток при тиреостатической терапии болезни Грейвса можно объяснить тем, что индуцированная тиамазолом клинико-лабораторная ремиссия болезни Грейвса способствует развитию адаптивных изменений в системе иммунитета уже на раннем этапе лечения заболевания, но не сопровождается полной иммунологической ремиссией. Отсутствие взаимосвязей между показателями тиреоидного статуса, уровнем антител к рТТГ и содержанием хелперных и регуляторных Т-лимфоцитов может быть связано с перекрестными интратиреоидными антигенными стимулами, существованием различных вариантов сплайсинга рТТГ, индуцированной тиамазолом смены титра 
преобладающих блокирующих или стимулирующих антител к рТТГ и соответствующей трансформацией аутоиммунной реакции [2, 3].

Таким образом, длительная консервативная терапия тиамазолом способствует повышению уровня регуляторных Т-лимфоцитов у пациентов с болезнью Грейвса, но не влияет на органоспецифические и генерализованные нарушения их супрессорной функции, причем эти нарушения не зависят от продолжительности эутиреоидного состояния, титра антител к рТТГ и, вероятно, могут поддерживать, и усиливать патологический процесс.

\section{Сопоставление с другими публикациями}

Результаты нашего исследования согласуются с работами авторов, которым удалось показать, что влияние высоких доз тиреостатиков на ряд иммунологических параметров не отличается от действия низких доз этих лекарственных средств, а основным фактором, от которого зависят иммунологические показатели, является гипертиреоз [14]. Такое влияние тиреостатических препаратов на клетки щитовидной железы, нормализующее ее функцию и восстанавливающее эутиреоз, не объясняет механизм персистенции иммунной реакции, хотя и препятствует стимулирующему действию ТТГ. В нескольких исследованиях сообщалось, что фенотипический состав лимфоцитов периферической крови отражает аутоиммунитет в щитовидной железе как выражение генерализованной активации иммунной системы у пациентов с гипертиреозом аутоиммунного генеза [8, 15]. Позднее было показано, что тиреоидные антигены активируют цитотоксические Т-клетки $\left(\mathrm{CD}^{+} \mathrm{CD}^{+}\right)$пациентов с болезнью Грейвса значительно слабее, чем соответствующие клетки здоровых доноров [6]. Кроме того, в более ранних работах сообщалось, что антиген рТТГ гораздо слабее активирует $\mathrm{CD}^{+} \mathrm{CD}^{+}{ }^{-}$-клетки пациентов с болезнью Грейвса, чем $\mathrm{CD}^{+} \mathrm{CD}^{+}$-лимфоциты здоровых лиц, больных хроническим аутоиммунным тиреоидитом, нетоксическим зобом и сахарным диабетом 1 типа [16].

Сохраняющиеся изменения в фенотипическом составе Т-лимфоцитов, несмотря на поддержание эутиреоидного состояния при лечении тиамазолом более 12 мес, которые были показаны в настоящем исследовании, позволяют утверждать, что высокий уровень $\mathrm{CD} 3^{+} \mathrm{CD} 4^{+} \mathrm{CD} 25^{+}$в периферической крови пациентов с болезнью Грейвса может способствовать миграции Th1-клеток из периферической крови в пораженную щитовидную железу. Что, в свою очередь, приводит к усилению презентации тиреоидных антигенов, способствуя дальнейшей активации Т-хелперов. При этом субпопуляция $\mathrm{CD}^{+} \mathrm{CD}^{+}$может быть источником продукции ИФН-ү, который является негативным регулятором гемопоэза и отражает преобладание Th1-, а не Th2-типа иммунного ответа в начальной фазе болезни Грейвса [12]. Полученные данные существенно дополняют результаты исследования соотношения хемокинов CXCR3/CCR4, указывающего на баланс Th1/Th2, в котором было показано значительное снижение вышеуказанного соотношения после лечения метимазолом по сравнению с исходным уровнем, причем соотношение было значительно ниже на 24-й, чем на 12-й неделе лечения [17]. Авторы делают вывод о том, что при лечении тиреостатическими препаратами происходит постепенный переход от доминиро- вания Th1 к доминированию Th2 иммунного ответа. Ряд современных данных указывает на первичный дефект в снижении количества и функции неспецифических регуляторных Т-лимфоцитов в гипертиреоидной фазе болезни Грейвса, преодоление которого происходит по мере развития медикаментозного эутиреоза и нормализации соотношения Th1/Th2.

Многие авторы подчеркивают, что в основе рецидива гипертиреоза при болезни Грейвса лежит частичное сохранение нарушенной генерализованной и антигенспецифичной супрессорной функции регуляторных Т-лимфоцитов, которая не зависит от гипер-, гипо- или эутиреоидного состояния больных $[5,18]$. Более раннее сравнительное исследование с использованием иммуногистохимии аспиратов щитовидной железы пациентов с болезнью Грейвса и образцов здоровой ткани показало, что антитиреоидные препараты могут усиливать экспрессию Fas-лиганда (FasL) на мембране тиреоцитов с последующей активацией Fas-индуцированного апоптоза этих клеток [19]. Причем было обнаружено, что обработанные в условиях in vitro метимазолом тиреоциты индуцировали FasL-зависимый апоптоз в культивируемых лимфоцитах, в то время как обработка метимазолом лимфоцитов, выращенных в отсутствие тиреоцитов, не оказывала такого эффекта. Таким образом, FasL высоко экспрессируется в фолликулярных клетках щитовидной железы у пациентов с болезнью Грейвса, получающих тиреостатические препараты, и может способствовать прямому иммуномодулирующему эффекту тионамидов.

Современные представления свидетельствуют о том, что система Fas-FasL является наиболее изученной системой активации гибели клеток в ходе терминации иммунного ответа, а дисрегуляция рецептор-опосредованного апоптоза играет ключевую роль в иммунопатогенезе болезни Грейвса [20]. Настоящее исследование существенно дополняет представления о развитии адаптивного иммунитета при болезни Грейвса на фоне медикаментозного лечения тиамазолом. Было показано, что при приеме тиамазола пациентами с болезнью Грейвса повышение числа Т-клеток, несущих маркер ранней активации $\left(C D 25^{+}\right)$, наблюдается уже в 1-й фазе терапии и может отражать потенциальный механизм генерализованной иммунологической толерантности тиреоцитов от атаки Т-лимфоцитами. Этот факт приводит к утверждению важной роли Fas-опосредованного апоптоза не только интратиреоидных лимфоцитов, но и периферических аутореактивных Т-лимфоцитов при болезни Грейвса.

\section{Клиническая значимость результатов}

Выявленные закономерности в изменении фенотипического состава Т-лимфоцитов периферической крови на фоне тиреостатической терапии свидетельствуют о возможных иммуномодулирующих эффектах длительной терапии тиамазолом на систему адаптивного иммунитета при болезни Грейвса. Полученные результаты позволяют выделить субпопуляцию Т-регуляторных клеток $\left(\mathrm{CD} 3^{+} \mathrm{CD} 4^{+} \mathrm{CD} 127^{\text {Low }} \mathrm{CD} 25^{\text {High }}\right)$ не только как мишень для этиотропной терапии супрессорного дефекта иммунной системы, но и в качестве возможного маркера иммунологической ремиссии заболевания перед завершением курса длительной тиреостатической терапии в клинической практике. 


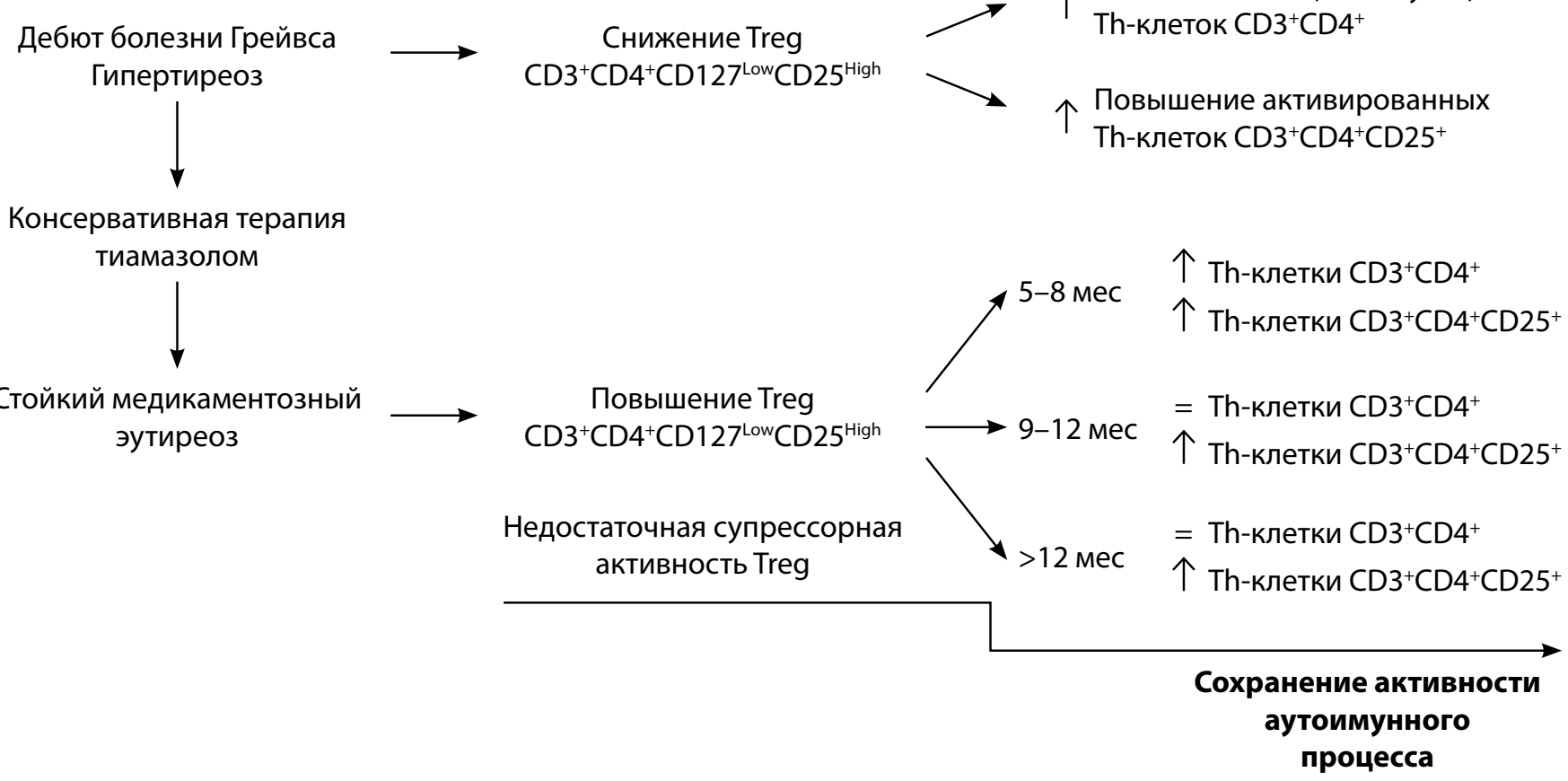

Рис. 1. Изменение соотношения Th и Treg в периферической крови у пациентов с болезнью Грейвса в зависимости от продолжительности медикаментозного эутиреоза при консервативной терапии тиамазолом.

Примечание. Стрелками указаны изменения относительно контрольных значений. "=" - соответствие контрольному диапазону.

\section{Ограничения исследования}

К возможным ограничениям настоящего исследования популяции пациентов с болезнью Грейвса можно отнести иммуногенетические аспекты уже имеющихся нарушений в иммунной системе, а также генетические аномалии тиреоцитов. Клиническая значимость иммуномодулирующего эффекта тиамазола может быть смещена в зависимости от силы дефекта антигеноспецифической иммуносупрессии в сочетании с неспецифическим влиянием факторов внешней среды в каждом конкретном случае заболевания.

\section{Направления дальнейших исследований}

Необходимы дополнительные иммуногенетические исследования для персонализированного стратегического планирования длительной консервативной терапии тиамазолом в зависимости от ассоциации экспрессии HLA-DR и специфического дефекта иммунорегуляции, обусловленного аномалией генов, кодирующих антигенпрезентирующие молекулы. Перспективными представляются исследования по изучению хемилюминесцентной активности и внутриклеточного метаболизма клеток иммунной системы в аспекте ингибирования образования свободных кислородных радикалов при длительной тиреостатической терапии, которые не только дополнят фундаментальные аспекты состояния клеточноопосредованного иммунитета при болезни Грейвса, но и помогут определить терапевтические мишени для этиотропной терапии.

\section{ЗАКЛЮЧЕНИЕ}

Проведено клинико-иммунологическое обследование пациентов с болезнью Грейвса в зависимости от продолжительности стойкого медикаментозного эутиреоза.
Охарактеризованы изменения в фенотипическом составе Т-лимфоцитов периферической крови с учетом компенсации гипертиреоза и уровня антител к рТТГ. Установлены механизмы адаптационных изменений в иммунной системе больных болезнью Грейвса при длительной тиреостатической терапии, независимо от титра циркулирующих антител к рТТГ и продолжительности эутиреоидного состояния. В зависимости от продолжительности медикаментозного эутиреоза растет популяция регуляторных Т-лимфоцитов с фенотипом $\mathrm{CD}^{+}{ }^{+} \mathrm{CD} 4^{+} \mathrm{CD} 127^{\text {Low }} \mathrm{CD} 25^{\text {High }}$ как в группе больных со стойким медикаментозным эутиреозом от 5 до 8 мес, так и у пациентов с длительностью эутиреоза от 9 до 12 мес. Повышение количества активированных T-хелперов с фенотипом $\mathrm{CD}^{+} \mathrm{CD}^{+}{ }^{+} \mathrm{CD} 25^{+}$ сохраняется у пациентов с длительностью медикаментозного эутиреоза более года. При болезни Грейвса с продолжительностью медикаментозного эутиреоза более 12 мес отмечается компенсаторное повышение регуляторных Т-лимфоцитов, а общее количество Т-хелперов восстанавливается до уровня контроля. Количество Treg в периферической крови у пациентов с болезнью Грейвса повышается с увеличением длительности медикаментозного эутиреоза, но, несмотря на увеличение регуляторных Т-лимфоцитов, процент активированных Th-клеток остается высоким независимо от продолжительности эутиреоидного состояния при консервативном лечении тиамазолом (рис. 1).

Выявленные изменения в фенотипическом составе Т-лимфоцитов при длительном лечении тиамазолом демонстрируют необходимость разработки таргетной терапии, направленной на восстановление дефекта неспецифических и антигеноспецифических регуляторных Т-лимфоцитов, вследствие которого супрессорная функция регуляторных Т-клеток при болезни Грейвса активируется в меньшей степени. 


\section{ДОПОЛНИТЕЛЬНАЯ ИНФОРМАЦИЯ}

Согласие пациентов. Все исследования выполнены с информированного согласия испытуемых и в соответствии с Хельсинкской декларацией Всемирной ассоциации «Этические принципы проведения научных медицинских исследований с участием человека» с поправками 2013 г. и «Правилами клинической практики в Российской Федерации», утвержденными Приказом Минздрава РФ от 19.06.2003 г. № 266.

Источники финансирования. Исследование выполнялось на базе лаборатории молекулярно-клеточной физиологии и патологии
Федерального исследовательского центра «Красноярский научный центр Сибирского отделения Российской академии наук», обособленное подразделение «НИИ медицинских проблем Севера».

Конфликт интересов. Авторы декларируют отсутствие явных и потенциальных конфликтов интересов, связанных с содержанием настоящей статьи.

Участие авторов. Все авторы одобрили финальную версию статьи перед публикацией, выразили согласие нести ответственность за все аспекты работы, подразумевающую надлежащее изучение и решение вопросов, связанных с точностью или добросовестностью любой части работы.

\section{СПИСОК ЛИТЕРАТУРЫ| REFERENCES}

1. Geng L, Yang J, Tang X, et al. SLAM/SAP Decreased Follicular Regulatory T Cells in Patients with Graves'Disease. J Immunol Res [Internet]. 2021 Apr [cited 2021 Apr 19]: [about 11 p.]. Available from: https://www.ncbi.nlm.nih.gov/pmc/articles/PMC8079219/pdf/ JIR2021-5548463.pdf

2. García-Mayor RV, Álvarez-Vázquez P, Fluiters E, et al. Longterm remission following antithyroid drug withdrawal in patients with Graves' hyperthyroidism: parameters with prognostic value. Endocrine. 2019;63(2):316-322. doi: https://doi.org/10.1007/s12020-018-1785-z

3. Yu W, Wu N, Li L, et al. Side effects of PTU and MMI in the treatment of hyperthyroidism: a systematic review and meta-analysis. Endocr Pract. 2020;26(2):207-217. doi: https://doi.org/10.4158/EP-2019-0221

4. Park S, Song E, Oh HS, et al. When should antithyroid drug therapy to reduce the relapse rate of hyperthyroidism in Graves' disease be discontinued? Endocrine. 2019;65(2):348-356. doi: https://doi.org/10.1007/s12020-019-01987-w

5. Park SY, Kim BH, Kim M, et al. The longer the antithyroid drug is used, the lower the relapse rate in Graves' disease: a retrospective multicenter cohort study in Korea. Endocrine [Internet]. $2021 \mathrm{Apr}$ [cited 2021 Apr 15]: [about 8p.]. Available from: https://link.springer. com/content/pdf/10.1007/s12020-021-02725-x.pdf

6. Chen Z, Liu Y, Hu S, Zhang M, et al. Decreased Treg Cell and TCR Expansion Are Involved in Long-Lasting Graves' Disease. Front Endocrinol (Lausanne) [Internet]. 2021 Apr [cited 2021 Apr 12]: [about 12p.]. Available from: https://www.frontiersin.org/ articles/10.3389/fendo.2021.632492/full

7. https://rae-org.ru/ [Интернет]. Российская Ассоциация Эндокринологов. Доступно по: https://rae-org.ru/system/files/ documents/pdf/kr_versiya_3.0_fin_otredaktirovan_16.05.2021g.pdf. Ссылка активна на 16.05.2021.

8. De Leo S, Lee SY, Braverman LE. Hyperthyroidism. Lancet. 2016;388(10047):906-918. doi: https://doi.org/10.1016/S0140-6736(16)00278-6

9. Shyamasunder AH, Abraham P. Measuring TSH receptor antibody to influence treatment choices in Graves' disease. Clin Endocrinol (Oxf). 2017;86(5):652-657. doi: https://doi.org/10.1111/cen.13327

10. Zhang $X$, Olsen N, Zheng SG. The progress and prospect of regulatory $T$ cells in autoimmune diseases. J Autoimmun. 2020;111:102-461. doi: https://doi.org/10.1016/j.jaut.2020.102461

11. Granito A, Muratori L, Lalanne C, et al. Hepatocellular carcinoma in viral and autoimmune liver diseases: Role of CD4+ CD25+ Foxp3+ regulatory T cells in the immune microenvironment. World J Gastroenterol. 2021;27(22):2994-3009. doi: https://doi.org/10.3748/wjg.v27.i22.2994
12. Li Q, Wang B, Mu K, et al. The pathogenesis of thyroid autoimmune diseases: New T lymphocytes - Cytokines circuits beyond the Th1-Th2 paradigm. J Cell Physiol. 2019;234(3):2204-2216. doi: https://doi.org/10.1002/jcp.27180

13. Савченко А.А., Дудина М.А., Борисов А.Г., и др. Влияние хелперных и регуляторных Т-клеток на фенотипический состав В-лимфоцитов крови и щитовидной железы при болезни Грейвс а. // Медицинская иммунология. - 2018. - Т. 20. №3. - C. 431-438. [Savchenko AA, Dudina MA, Borisov AG, et al. Effects of helper and regulatory cells on phenotypic composition of blood B lymphocytes and thyroid gland in Graves' disease. Meditsinskaya Immunologiya. 2018;20(3):431-438. (In Russ.)]. doi: https://doi.org/10.15789/1563-0625-2018-3-431-438

14. Langenstein C, Schork D, Badenhoop K, et al. Relapse prediction in Graves' disease: Towards mathematical modeling of clinical, immune and genetic markers. Rev Endocr Metab Disord. 2016;17(4):571-581. doi: https://doi.org/10.1007/s11154-016-9386-8

15. Gallo D, Piantanida E, Gallazzi M, et al. Immunological Drivers in Graves' Disease: NK Cells as a Master Switcher. Front Endocrinol (Lausanne) [Internet]. 2020 Jul [cited 2020 Jul 17]; 11(406): [about 10 p.]. Available from: https://www.frontiersin.org/articles/10.3389/fendo.2020.00406/full

16. Mukuta T, Nishikawa M, Arreaza G, et al. The effect of adding a surfeit of autologous $C D 8^{+} T$ cells to scid mice after secondary rexenografts of Graves' thyroid tissue. Thyroid. 1996;6(5):429-436. doi: https://doi.org/10.1089/thy.1996.6.429

17. Inukai Y, Momobayashi A, Sugawara N, et al. Changes in expression of T-helper (Th) 1-and Th2-associated chemokine receptors on peripheral blood lymphocytes and plasma concentrations of their ligands, interferon-inducible protein-10 and thymus and activation-regulated chemokine, after antithyroid drug administration in hyperthyroid patients with Graves' disease. Eur J Endocrinol. 2007;156(6):623-630. doi: https://doi.org/10.1530/EJE-07-0019

18. Werner SC, Ingbar SH. Treatment of Thyrotoxicosis. In: Braverman LE, Utiger RD, editors. The Thyroid: A Fundamental and Clinical Text. Philadelphia: Lippincott Williams \& Wilkins; 2005. P. 665-675.

19. Mitsiades N, Poulaki V, Tseleni-Balafouta S, et al. Fas ligand expression in thyroid follicular cells from patients with thionamide-treated Graves' disease. Thyroid. 2000;10(7):527-532. doi: https://doi.org/10.1089/thy.2000.10.527

20. Ferrari SM, Fallahi P, Elia G, et al. Novel therapies for thyroid autoimmune diseases: An update. Best Pract Res Clin Endocrinol Metab. 2020;34(1):101-366. doi: https://doi.org/10.1016/j.beem.2019.101366

Рукопись получена: 03.09.2021. Одобрена к публикации: 09.11.2021. Опубликована online: 31.12.2021.

ИНФОРМАЦИЯ ОБ АВТОРАХ [AUTHORS INFO]

* Дудина Маргарита Андреевна, к.м.н. [Margarita A. Dudina, MD, PhD]; адрес: 660022, Россия, Красноярск, ул. Партизана Железняка, д. 1 [address: 1 Zeleznyaka street, 660022 Krasnoyarsk, Russia]; ORCID: http://orcid.org/0000-0002-2776-927X; eLibrary SPIN: 4854-1926; e-mail: margo85_@bk.ru

Догадин Сергей Анатольевич, д.м.н., професcop [Sergey A. Dogadin, MD, PhD, Professor]; ORCID: http://orcid.org/0000-0002-1709-466X; eLibrary SPIN: 4803-3756; e-mail: sadogadin@gmail.com 
Савченко Андрей Анатольевич, д.м.н., професcop [Andrei A. Savchenko, MD, PhD, Professor];

ORCID: http://orcid.org/0000-0001-5829-672X; eLibrary SPIN: 3132-8260; e-mail: aasavchenko@yandex.ru

Беленюк Василий Дмитриевич [Vasiliy D. Belenyuk, MD]; ORCID: http://orcid.org/0000-0003-2848-0846;

eLibrary SPIN: 6195-6630; e-mail: dyh.88@mail.ru

\section{ЦИТИРОВАТЬ}

Дудина М.А., Догадин С.А., Савченко А.А., Беленюк В.Д. Фенотипический состав Т-лимфоцитов периферической крови у пациентов с болезнью Грейвса при длительной тиреостатической терапии тиамазолом // Проблемы эндокринологии. 2021. — T. 67. — №6. - C. 39-49. doi: https://doi.org/10.14341/probl12812

\section{TO CITE THIS ARTICLE}

Dudina MA, Dogadin SA, Savchenko AA, Belenyuk VD. T-lymphocytes phenotypic composition of peripheral blood in patients with Graves' disease undergoing conservative therapy with thiamazole. Problems of Endocrinology. 2021;67(6):39-49. doi: https://doi.org/10.14341/probl12812 